\title{
Antiferromagnetic sawtooth chain with Heisenberg and Ising bonds
}

\author{
V. Ohanyan \\ 1 Department of Theoretical Physics, Yerevan State University, Al. Manoogian 1, 0025 Yerevan, Armenia \\ 2 Yerevan Physics Institute, Alikhanian br. 2, 0036, Yerevan, Armenia
}

Received May 28, 2009, in final form June 18, 2009

The sawtooth chain with pairs of $S=1 / 2$ spins interacting with $X X Z$-interactions placed on each second tooth is considered. All other interaction bonds are taken to be of Ising type. Exact statistical mechanical solution of the model within the direct transfer-matrix technique is obtained. The solution allows one to obtain exact analytic expressions for all thermodynamic functions of the model. Ground state properties are also investigated, the corresponding ground state phase diagram is presented.

Key words: sawtooth chain, exact solutions, magnetization plateaus

PACS: $75.10 . P q$

\section{Introduction}

The sawtooth chain or delta chain is a one-dimensional lattice spin system with a topology of corner-sharing triangles (figure 1). This system is famous for a number of important features. Physically, magnetic lattices corresponding to the sawtooth chain are found in a number of compounds, in delafossite $\mathrm{YCuO}_{2.5}[1,2]$ and olivines with structures $\mathrm{ZnL}_{2} \mathrm{~S}_{4}(\mathrm{~L}=\mathrm{Er}, \mathrm{Tm}, \mathrm{Yb})[3]$ to cite a couple of examples. Antiferromagnetic Heisenberg model on sawtooth chain is strongly frustrated, however, in contrast to many other frustrated systems, the ground state of sawtooth chain is known exactly. Namely, the ground state in case of cyclic boundary conditions is a two-fold degenerated dimerised structure where either each left pair of spin at each triangle or each right pair forms spin-singlet states (dimers) [4]. The excitations of the sawtooth chain are gapped and are of topological origin. They are found to be "kink" - "antikink"-type domain wall structures $[5,6]$. Anisotropic variants of sawtooth chain have also been investigated revealing additional features $[7,8]$. Another important feature of the sawtooth chain as well as of some other frustrated spin and Hubbard lattices revealed quite recently is the appearance of localized magnon states (dispersionless excitation bands) [9-12]. The localized low energy excitations in sawtooth chain which exist due to frustrated geometry, more precisely, due to the triangular plaquette, affect the lowtemperature thermodynamics. The corresponding eigenstates have been constructed for sawtooth chain in case of Heisenberg model in [12] and for Hubbard electrons in [10]. Calculating the ground state degeneracies for the localized magnon (or electron) states one can obtain explicit expressions for thermodynamic quantities for low temperatures and near saturation field $[10,12]$. However, the problem of exact description of whole thermodynamics for sawtooth chain as well as for many other interacting quantum spin systems is still an open issue.

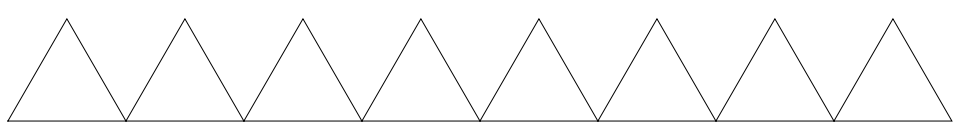

Figure 1. The sawtooth chain. 
One can mention a formal approach to these problem which is not justified properly yet but in some particular cases demonstrates rather good agreement with experimental data and numerical calculations. The approximation consists in replacement of some or all interaction bonds with Ising ones [13-24]. As a result one can obtain an interacting spin system which allows one to calculate all thermodynamic functions analytically. In this paper we use this approach for exact solution of the system with Ising and Heisenberg interaction bonds on sawtooth chain. To make possible exact calculation of the partition function within transfer-matrix method we consider all interaction bonds of sawtooth chain as Ising ones except left bonds on each second triangle. Spins connected by these bonds interact by Heisenberg $X X Z$ interaction. Such a construction allows one to represent the Hamiltonian in the form of sum of block Hamiltonian commutating to each other, thus, the exponent in partition function can be expanded to the product of exponents corresponding to each block. Due to this fact, the method of transfer-matrix can be exploited leading to an exact calculation of all thermodynamic functions. In the first section we formulate the model and give its exact solution, then, in the next section, we analyze various ground states of the system and draw a corresponding phase diagram. The last section contains concluding remarks.

\section{The model and its exact solution}

Let us consider the sawtooth chain in which the left pair of spins on every second triangle interacts with the $X X Z$-Heisenberg interaction, while all other interaction bonds are of Ising type, i.e. the interactions include only $z$-component of spin operators. The corresponding Hamiltonian

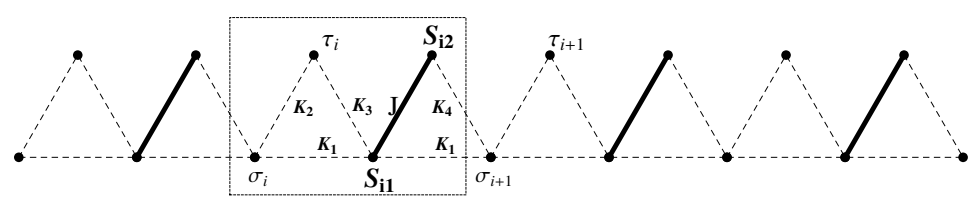

Figure 2. The sawtooth chain with Heisenberg bond on every second triangle. The Heisenberg bond is marked with bold line. Group of spins included into the dotted rectangle correspond to one block with Hamiltonian $\mathcal{H}_{i}$.

is suitable to be written as a sum of block Hamiltonians where each block contains 5 spins: a pair of spins connected with quantum bond, two adjacent spins from the basement and one spin at the top of the triangle from the left hand side (see figure 2):

$$
\begin{aligned}
\mathcal{H}= & \sum_{i=1}^{N}\left(\mathcal{H}_{i}+K_{2} \sigma_{i} \tau_{i}-H_{1} \tau_{i}-\frac{H_{2}}{2}\left(\sigma_{i}+\sigma_{i+1}\right)\right) \\
\mathcal{H}_{i}= & J\left(\Delta\left(S_{i 1}^{x} S_{i 2}^{x}+S_{i 1}^{y} S_{i 2}^{y}\right)+S_{i 1}^{z} S_{i 2}^{z}\right)+K_{1} S_{i 1}^{z}\left(\sigma_{i}+\sigma_{i+1}\right) \\
& +K_{3} S_{i 1}^{z} \tau_{i}+K_{4} S_{i 2}^{z} \sigma_{i+1}-H\left(S_{i 1}^{z}+S_{i 2}^{z}\right),
\end{aligned}
$$

where $S_{i, a}^{\alpha}, a=1,2, \alpha=x, y, z$ are $S=1 / 2$ spin operator components of the spin connected by quantum bond from $i-t h$ block, $\sigma_{i}$ and $\sigma_{i+1}$ are two adjacent spins from the basement and $\tau_{i}$ is the spin on the top of left triangle; as only $z$-components of all $\sigma$ and $\tau$ spins are included in the interaction, one can consider them just as a classical variable, taking values $\pm 1 / 2$. Here we also choose the most general form of interaction with different coupling constants $J, K_{1}, K_{2}, K_{3}, K_{4}$ for different kinds of bonds and different $g$-factors for Zeeman term corresponding to different kinds of spins. The partition function of the system then reads:

$$
\mathcal{Z}=\sum_{(\sigma)} \sum_{(\tau)} \operatorname{Sp}_{\mathbf{S}} \mathrm{e}^{-\beta \mathcal{H}} .
$$

Here one should sum over all values of classical variables $\sigma$ and $\tau$ and take a trace over all states of spin operators S. Due to commutativity of Hamiltonians for different blocks one can expand the 
exponent and get the product of the terms corresponding to different blocks. After that the traces for each block can be taken separately:

$$
\mathcal{Z}=\sum_{(\sigma)} \prod_{i=1}^{N} \sum_{\tau_{i}= \pm 1 / 2} \Omega\left(\sigma_{i}, \sigma_{i+1} \mid \tau_{i}\right) \mathrm{e}^{\beta H_{1} \tau_{i}-\beta K_{2} \tau_{i} \sigma_{i}+\beta \frac{H_{2}}{2}\left(\sigma_{i}+\sigma_{i+1}\right)}
$$

where

$$
\Omega\left(\sigma_{i}, \sigma_{i+1} \mid \tau_{i}\right)=\mathrm{Spe}^{-\beta \mathcal{H}_{i}}=\sum_{n=1}^{4} \mathrm{e}^{-\beta \lambda_{n}\left(\sigma_{i}, \sigma_{i+1} \mid \tau_{i}\right)}
$$

and $\lambda_{n}$ are four eigenvalues of the $\mathcal{H}_{i}$ :

$$
\begin{aligned}
& \lambda_{1,2}\left(\sigma_{i}, \sigma_{i+1} \mid \tau_{i}\right)=\frac{1}{4} J \mp \frac{1}{2}\left(2 H-K_{1}\left(\sigma_{i}+\sigma_{i+1}\right)-K_{3} \tau_{i}-K_{4} \sigma_{i+1}\right), \\
& \lambda_{3,4}\left(\sigma_{i}, \sigma_{i+1} \mid \tau_{i}\right)=-\frac{1}{4} J \mp \frac{1}{2} \sqrt{\left(K_{1}\left(\sigma_{i}+\sigma_{i+1}\right)+K_{3} \tau_{i}-K_{4} \sigma_{i+1}\right)^{2}+J^{2} \Delta^{2}}
\end{aligned}
$$

In equation (3) the sum over all states of spin $\tau$ in each block can be taken independently of the other, then yielding

$$
\begin{aligned}
& \mathcal{Z}=\sum_{(\sigma)} \prod_{i=1}^{N} Z\left(\sigma_{i}, \sigma_{i+1}\right) \mathrm{e}^{\beta \frac{H_{2}}{2}\left(\sigma_{i}+\sigma_{i+1}\right)}, \\
& Z\left(\sigma_{i}, \sigma_{i+1}\right)=\Omega\left(\sigma_{i}, \sigma_{i+1} \mid 1 / 2\right) \mathrm{e}^{\beta \frac{1}{2}\left(H_{1}-K_{2} \sigma_{i}\right)}+\Omega\left(\sigma_{i}, \sigma_{i+1} \mid-1 / 2\right) \mathrm{e}^{-\beta \frac{1}{2}\left(H_{1}-K_{2} \sigma_{i}\right)} .
\end{aligned}
$$

Thus, the partition function takes the form similar to that of the single chain with $N$ sites with classical variables $\sigma_{i}$ in them. The partition function can be calculated within the standard transfermatrix technique (see for example [25]):

$$
\mathcal{Z}=\sum_{(\sigma)} \prod_{i=1}^{N} T\left(\sigma_{i}, \sigma_{i+1}\right)=\operatorname{Tr}^{N}=\Lambda_{+}^{N}+\Lambda_{-}^{N}
$$

here the cyclic boundary conditions $\sigma_{i+N}=\sigma_{i}$ are imposed and 2 by 2 transfer-matrix $\mathbf{T}$ with eigenvalues $\Lambda_{ \pm}$has the following form:

$$
\mathbf{T}=\left(\begin{array}{cc}
Z(1 / 2,1 / 2) \mathrm{e}^{\beta \frac{H_{2}}{2}} & Z(1 / 2,-1 / 2) \\
Z(-1 / 2,1 / 2) & Z(-1 / 2,-1 / 2) \mathrm{e}^{-\beta \frac{H_{2}}{2}}
\end{array}\right)
$$

Thus, for free energy per one block of the sawtooth lattice with mixed Heisenberg and Ising bonds described above in the thermodynamic limit when only maximal eigenvalue survives from equation (7) one obtains

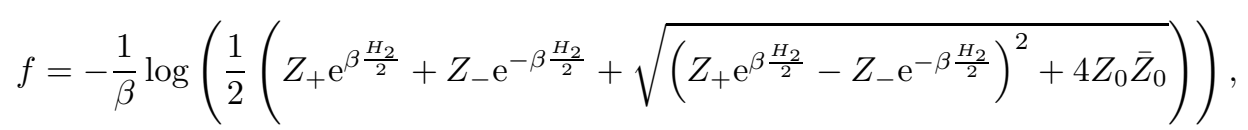

where the following notations are introduced: $Z_{+}=Z(1 / 2,1 / 2), Z_{-}=Z(-1 / 2,-1 / 2), Z_{0}=$ $Z(1 / 2,-1 / 2)$ and $\bar{Z}_{0}=Z(-1 / 2,1 / 2)$. Then, one can obtain analytic expressions for all thermodynamic quantities of the systems by taking derivatives of equation (9). Thus, equations (3)-(9) exactly solve the problem of thermodynamics of the systems under consideration. Let us finally list the explicit expressions for the entries of transfer-matrix for a particular choice of couplings, mentioned in the beginning of the next section, $K_{1}=J_{1}$ and $K_{2}=K_{3}=K_{4}=J$ and for the same 
value of $g$-factor for all spins, which imply $H_{1}=H_{2}$.

$$
\begin{aligned}
& Z_{+}=2\left(\mathrm{e}^{\beta \frac{1}{2}(H-1 / 2 J)}\left(\mathrm{e}^{-\beta \frac{J}{4}} \cosh \left(\beta\left(H-1 / 2\left(J_{1}-1 / 2 J\right)\right)\right)+\mathrm{e}^{\beta \frac{J}{4}} \cosh \left(\beta 1 / 2 \sqrt{J_{1}^{2}+J^{2} \Delta^{2}}\right)\right)\right. \\
&+\mathrm{e}^{-\beta \frac{1}{2}(H-1 / 2 J)}\left(\mathrm{e}^{-\beta \frac{J}{4}} \cosh \left(\beta\left(H-1 / 2\left(J_{1}-3 / 2 J\right)\right)\right)\right. \\
&\left.\left.+\mathrm{e}^{\beta \frac{J}{4}} \cosh \left(\beta 1 / 2 \sqrt{\left(J_{1}-J\right)^{2}+J^{2} \Delta^{2}}\right)\right)\right) \\
& Z_{-}=2\left(\mathrm{e}^{\beta \frac{1}{2}(H+1 / 2 J)}\left(\mathrm{e}^{-\beta \frac{J}{4}} \cosh \left(\beta\left(H+1 / 2\left(J_{1}-1 / 2 J\right)\right)\right)+\mathrm{e}^{\beta \frac{J}{4}} \cosh \left(\beta 1 / 2 \sqrt{\left(J_{1}-J\right)^{2}+J^{2} \Delta^{2}}\right)\right)\right. \\
&+\mathrm{e}^{-\beta \frac{1}{2}(H+1 / 2 J)}\left(\mathrm{e}^{-\beta \frac{J}{4}} \cosh \left(\beta\left(H+1 / 2\left(J_{1}+3 / 2 J\right)\right)\right)\right. \\
&\left.\left.+\mathrm{e}^{\beta \frac{J}{4}} \cosh \left(\beta 1 / 2 \sqrt{J_{1}^{2}+J^{2} \Delta^{2}}\right)\right)\right) \\
& 2\left(\mathrm{e}^{\beta \frac{1}{2}(H-1 / 2 J)}\left(\mathrm{e}^{-\beta \frac{J}{4}} \cosh (\beta(H-1 / 4 J))\right)+\mathrm{e}^{\beta \frac{J}{4}} \cosh \left(\beta 1 / 2 J \sqrt{1+\Delta^{2}}\right)\right) \\
&\left.+\mathrm{e}^{-\beta \frac{1}{2}(H-1 / 2 J)}\left(\mathrm{e}^{-\beta \frac{J}{4}} \cosh (\beta(H+3 / 4 J))+\mathrm{e}^{\beta \frac{J}{4}} \cosh (\beta 1 / 2 J \Delta)\right)\right) \\
&\left.Z_{0}\right)\left(\mathrm{e}^{\beta \frac{1}{2}(H+1 / 2 J)}\left(\mathrm{e}^{-\beta \frac{J}{4}} \cosh (\beta(H-3 / 4 J))\right)+\mathrm{e}^{\beta \frac{J}{4}} \cosh (\beta 1 / 2 J \Delta)\right) \\
&\left.+\mathrm{e}^{-\beta \frac{1}{2}(H+1 / 2 J)}\left(\mathrm{e}^{-\beta \frac{J}{4}} \cosh (\beta(H+1 / 4 J))+\mathrm{e}^{\beta \frac{J}{4}} \cosh \left(\beta 1 / 2 J \sqrt{1+\Delta^{2}}\right)\right)\right) \\
&\left.\bar{Z}_{0}=\right)
\end{aligned}
$$

\section{Ground states phase diagram for antiferromagnetic couplings}

Let us analyze different ground states of the model. For the sake of simplicity we restrict ourselves to the case of only two different coupling constants, one along the basement $J_{1}$ and another one between spins on the top and spins on the basement $J$. This means one should put $K_{1}=J_{1}$ and $K_{2}=K_{3}=K_{4}=J$. We also consider only antiferromagnetic coupling $J>0, J_{1}>0$, the rest cases are to some extent trivial ones. Solving the eigenvalues and eigenvectors problem for block Hamiltonian $\mathcal{H}_{i}$ and taking into account all combinations of spins $\sigma_{i}, \sigma_{i+1}$ and $\tau_{i}$, one can get the following periodic eigenstates for the whole chain with period equal to the period of the lattice up to the inversion of all spins: three antiferromagnetic states with $M=0$, two of which are degenerated and differ from each other by the flip of all $\sigma$ and $\tau$ spins(the corresponding energies per one block are presented)

$$
\begin{aligned}
\left|A M_{+}\right\rangle & =\prod_{i=1}^{N}\left|S^{z}=0,+\right\rangle_{i} \bigotimes\left|\sigma_{i}=\uparrow, \tau_{i}=\downarrow\right\rangle \\
\left|A M_{-}\right\rangle \prod_{i=1}^{N} & =\left|S^{z}=0,-\right\rangle_{i} \bigotimes\left|\sigma_{i}=\downarrow, \tau_{i}=\uparrow\right\rangle \\
\epsilon_{A M} & =-\frac{1}{2}\left(J+\sqrt{\left(J-J_{1}\right)^{2}+J^{2} \Delta^{2}}\right)
\end{aligned}
$$

where $\left|S^{z}=0, \pm\right\rangle_{i}$ stands for the following state of two $\mathbf{S}$ spins form $i$-th block with total projection equal to zero

$$
\begin{aligned}
\left|S^{z}=0, \pm\right\rangle & =\frac{1}{\sqrt{1+\gamma_{ \pm}^{2}}}\left(|\uparrow \downarrow\rangle-\gamma_{ \pm}|\downarrow \uparrow\rangle\right) \\
\gamma_{ \pm} & =\frac{ \pm\left(J-J_{1}\right)+\sqrt{\left(J-J_{1}\right)^{2}+J^{2} \Delta^{2}}}{J \Delta}
\end{aligned}
$$


This eigenstate corresponds to the $\lambda_{3}$ eigenvalue from equation (5). For arbitrary values of $\sigma$ and $\tau$ surrounding pairs of $\mathbf{S}$ spins, coefficient $\gamma$ from equation (12) is

$\gamma\left(\sigma_{i}, \sigma_{i+1} \mid \tau_{i}\right)=\frac{J\left(\sigma_{i+1}-\tau_{i}\right)-J_{1}\left(\sigma_{i}+\sigma_{i+1}\right)+\sqrt{\left(J\left(\sigma_{i+1}-\tau_{i}\right)-J_{1}\left(\sigma_{i}+\sigma_{i+1}\right)\right)^{2}+J^{2} \Delta^{2}}}{J \Delta}$.

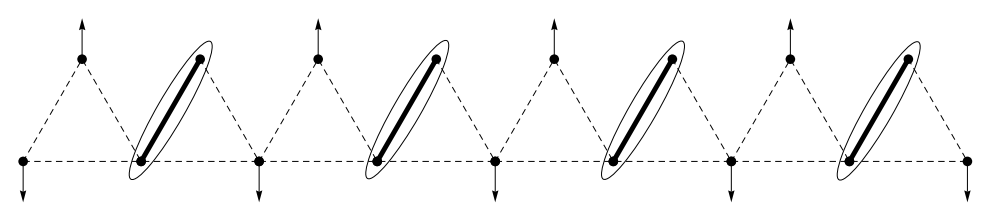

Figure 3. Spin configuration corresponding to the $\left|A M_{-}\right\rangle$antiferromagnetic state. Ovals mark the pairs of spin connected with Heisenberg bonds in $\left|S^{z}=0,-\right\rangle$ state. One should inverse all arrows to obtain $\left|S^{z}=0,+\right\rangle$ state.

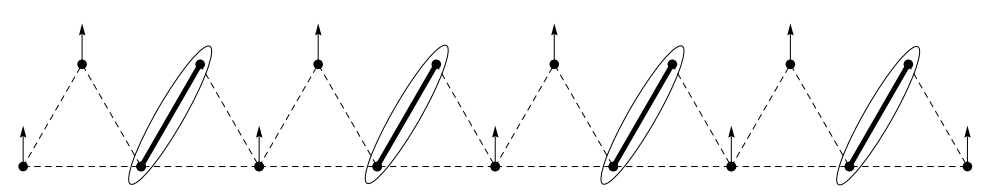

Figure 4. Spin configuration corresponding to the $|F 1\rangle$ ferrimagnetic state.

Thus, in these configurations the pair of spins connected with the Ising bonds are aligned opposite to each other, while each pair of spins connected with the Heisenberg bond is in a special state with $S_{\mathrm{tot}}^{z}=0$, see figure (3). Another antiferromagnetic ground state consists of triplet spin configurations on each Heisenberg bond and pairs of spins connected with Ising bond both pointed opposite to the direction of $S=1$ pairs. However, this ground state is never realized at $T=0$ for antiferromagnetic region of values of $J$ and $J_{1}$. There are also three ferrimagnetic states of the chain when external magnetic field is turned on. All these states have spatial period corresponding to the period of the chain and magnetization equal to $1 / 2$. In the first ferrimagnetic state all pairs of $\mathbf{S}$ spins are in a singlet state, while the rest $\sigma$ and $\tau$ spins are pointed along the field (figure 4):

$$
|F 1\rangle=\prod_{i=1}^{N}\left|S^{z}=0\right\rangle_{i} \bigotimes\left|\sigma_{i}=\uparrow, \tau_{i}=\uparrow\right\rangle, \quad \epsilon_{F 1}=-\frac{1}{2} \sqrt{J_{1}^{2}+J^{2} \Delta^{2}}-H,
$$

where eigenstate $\left|S^{z}=0\right\rangle_{i}$ is given by the same formula as equation (12) but with another value of the coefficient gamma, which is given by equation (13)

$$
\gamma=\frac{-J_{1}+\sqrt{J_{1}^{2}+J^{2} \Delta^{2}}}{J \Delta} .
$$

In the rest two ferrimagnetic states all pairs of $\mathbf{S}$ spins are in the triplet polarized state with $S^{z}=1$, while $\sigma$ and $\tau$ spins surrounding them are pointed opposite to each other:

$$
\begin{aligned}
|F 2\rangle & =\prod_{i=1}^{N}|\uparrow \uparrow\rangle_{i} \bigotimes\left|\sigma_{i}=\uparrow, \tau_{i}=\downarrow\right\rangle, \quad \epsilon_{F 2}=\frac{1}{4} J_{1}-H, \\
|F 3\rangle & =\prod_{i=1}^{N}|\uparrow \uparrow \uparrow\rangle_{i} \bigotimes\left|\sigma_{i}=\downarrow, \tau_{i}=\uparrow\right\rangle, \quad \epsilon_{F 3}=-\frac{1}{4} J_{1}-H .
\end{aligned}
$$


And finally in the spin polarized phase all spins are pointed along the field,

$$
|S P\rangle=\prod_{i=1}^{N}|\uparrow \uparrow\rangle_{i} \bigotimes\left|\sigma_{i}=\uparrow, \tau_{i}=\uparrow\right\rangle, \quad \epsilon_{S P}=J+\frac{1}{2} J_{1}-2 H
$$

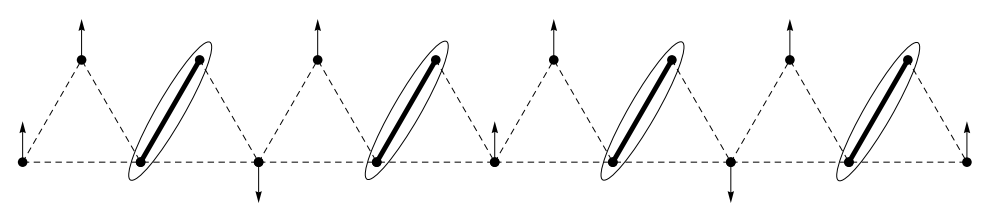

Figure 5. Spin configuration for corresponding to $|S M\rangle$ phase.

Besides the states with non-broken lattice symmetry the system under consideration can possess another ground state with a spatial period equal to two blocks. This state can be realized at $T=0$ only for $\eta=\frac{J_{1}}{J} \leqslant 1$. This state can be obtained from $\left|A M_{-}\right\rangle$by flipping every second $\sigma$ spin in the basement of the chain (figure 5). This state with broken translational symmetry corresponds to magnetization of the system equal to $1 / 4$. We will refer to it as a spin modulated phase, $|S M\rangle$. The spatial period of the state and the value of magnetization per site are in full agreement with Oshikawa-Yamanaka-Affleck criterion [26]. The corresponding energy per one block is

$$
\epsilon_{S M}=-\frac{1}{4} J\left(1+|\Delta|+\sqrt{1+\Delta^{2}}\right)-\frac{1}{2} H .
$$

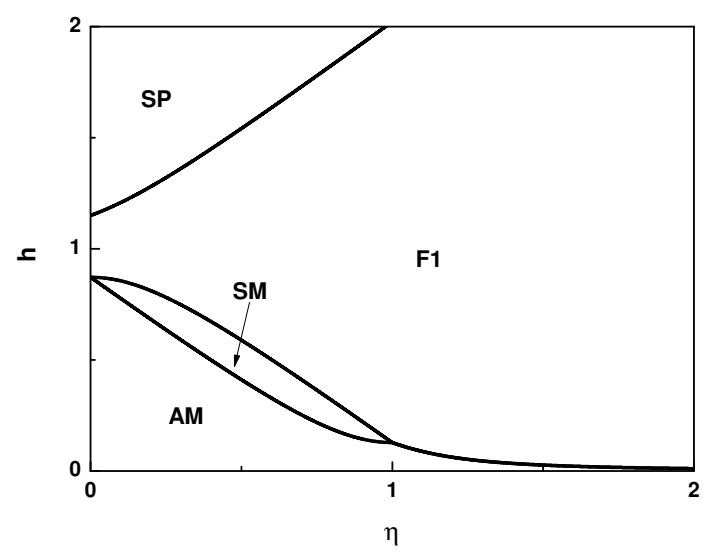

Figure 6. $T=0$ ground state phase diagram for the sawtooth chain with Ising and Heisenberg bonds for $J>0, J_{1}>0$ in $(\eta, h)$-plane for $\Delta=0.3$.

Now it is straightforward to draw the $T=0$ ground state phase diagram for antiferromagnetic couplings $J>0, J_{1}>0$. In figure (6) one can see the phase boundaries in $(\eta, h)$ plane $(h=H / J)$ for $\Delta=0.3$. An interesting feature of the system is that at antiferromagnetic values of couplings with the ground state for $H=0$ is unaffected by the value of $\eta$. Thus, the unique ground state is either $\left|A M_{-}\right\rangle$or $\left|A M_{+}\right\rangle$. One should distinguish two regions of $\eta$, namely, for $\eta<1$ increasing the magnetic field one can see transition to spin modulated phase with $M=1 / 4$ prior to the appearance of the ferrimagnetic structure $F 1$ with $M=1 / 2$. However, for $\eta>1$, antiferromagnetic phase changes immediately with the ferrimagnetic one, though the width of the $A M$ phase region (the value of the $h$ at which the zero temperature quantum phase transition takes the place) with $\eta \rightarrow \infty$ asymptotically goes to zero. So, one can see a small closed region of $S M$ phase in the 
$(\eta, h)$ plane. At the values $\eta=1, h=0$, the system demonstrates an additional feature, i. e., the macroscopic degeneracy of the ground state. At this point the $\mathbf{S}$ spins in each block are in the singlet state, whereas its adjacent $\sigma$ and $\tau$ can take an arbitrary value with one restriction $\sigma+\tau=0$. Thus, in each block, $\sigma$ and $\tau$ can freely take either $\sigma=1 / 2, \tau=-1 / 2$ or $\sigma=-1 / 2, \tau=1 / 2$ values. Thus, one obtains macroscopic two-fold degeneracy at $J=J_{1}$. One can refer to this state as a frustrated antiferromagnetic state:

$$
|F R\rangle=\prod_{i=1}^{N}\left|S^{z}=0\right\rangle_{i} \bigotimes\left|\xi_{i}\right\rangle,
$$

where $\left|\xi_{i}\right\rangle$ can be either $\left|\sigma_{i}=\uparrow, \tau_{i}=\downarrow\right\rangle$ or $\left|\sigma_{i}=\downarrow, \tau_{i}=\uparrow\right\rangle$ And finally the equations of phase boundaries between four phases presented in the phase diagram in figure (5) are listed below:

$$
\begin{array}{cllll}
\text { between }|A M\rangle \text { and } & |S M\rangle: & h=\frac{1}{2}\left(1-|\Delta|-\sqrt{1+\Delta^{2}}\right)+\sqrt{(1-\eta)^{2}+\Delta^{2}}, \\
\text { between }|A M\rangle \text { and }|F 1\rangle: & h=\frac{1}{2}\left(1+\sqrt{(1-\eta)^{2}+\Delta^{2}}-\sqrt{\eta^{2}+\Delta^{2}}\right), \\
\text { between }|S M\rangle \text { and }|F 1\rangle: \quad h=\frac{1}{2}\left(1+|\Delta|+\sqrt{1+\Delta^{2}}\right)-\sqrt{\eta^{2}+\Delta^{2}}, \\
\text { between }|F 1\rangle \text { and }|S P\rangle: \quad h=1+\frac{1}{2}\left(\eta+\sqrt{\eta^{2}+\Delta^{2}}\right) .
\end{array}
$$

\section{Conclusion}

In this paper using the example of sawtooth chain we considered the possibility to obtain exact thermodynamic solution for the one-dimensional quantum spin systems by considering their counterparts where some interaction bonds are changed with the Ising ones. Namely, we considered

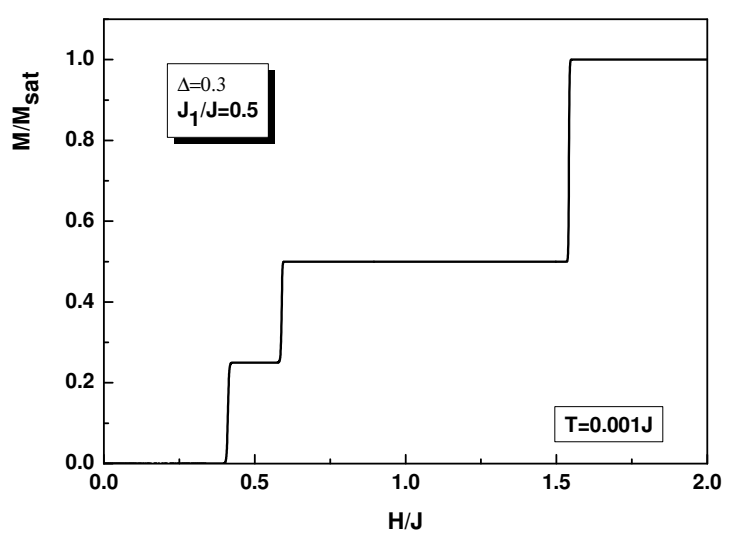

Figure 7. Magnetization process for $\eta=0.5$ at extremely low temperature $T=0.001 \mathrm{~J}$ and $\Delta=0.3$. Three magnetization plateaus at $M=0, M=1 / 4$ and $M=1 / 2$ correspond to $A M$, $S M$ and $F 1$ phases.

the pairs of $S=1 / 2$ quantum spins interacting with $X X Z$ interaction arranged in the sawtooth chain in such a way which allows one to use transfer-matrix technique for exact calculations. This result continues the series of investigations of the subject performed earlier for other onedimensional spin systems with Ising and Heisenberg bonds [13-24]. These results are not only of academic interest. There are many one-dimensional spin systems which are the models of a real magnetic material and which are not integrable. Until now, the only truly reliable way of describing 
the thermodynamic and magnetic properties of such a system is laborious numerical calculations. However, the results of recent investigations provide evidence that changing some bonds of the system with Ising ones, on the one hand, makes the thermodynamical problem exactly solvable and, on the other hand, does not drastically change the magnetic properties of the system at least in case when only ferromagnetic bounds have been changed. One can look at the plots of low-temperature magnetization curves for the system considered in the paper (figures $(7)$, (8)). As it can be seen from phase diagram (figure 6), there is qualitative difference between $\eta<0$ and

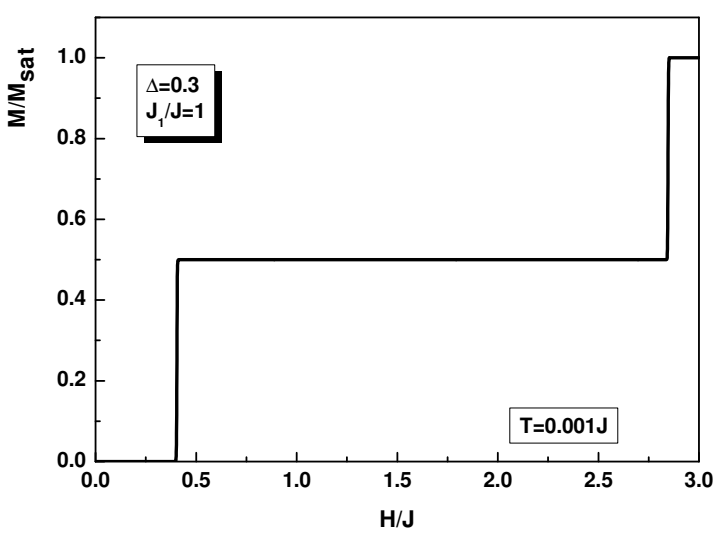

Figure 8. Magnetization process for $\eta=1$ at extremely low temperature $T=0.001 J$ and $\Delta=0.3$. Spin modulated phase corresponding to the plateau at $M=1 / 4$ is absents here. One can see only two plateaus at $M=0$ and $M=1 / 2$ corresponding to $F R$ and $F 1$ phases.

$\eta \geqslant 1$ cases. For $\eta<1$, one obtains the curve with three intermediate magnetization plateaus, at $M=0, M=1 / 4$ and $M=1 / 2$, which corresponds to the stability regions of $A M, S M$ and $F 1$ structures, respectively. In figure (7) one can see the corresponding plot for $\Delta=0.3$ and $\eta=0.5$. For $\eta=1$ and $\Delta=0.3$ one can see in figure (8) only one plateau at $M=1 / 2$. This result qualitatively corresponds to that obtained in purely quantum sawtooth chain [12]. Numerically obtained magnetization curves for sawtooth Heisenberg chain contain only plateaus at $M=0$ and $M=1 / 2$ for the whole range of parameters. Though there is a rather large discrepancy in quantitative characteristics, such as positions of the terminal points of the plateau, this can be explained by the fact that in our case we have changed antiferromagnetic bonds with Ising ones, which generally speaking makes much more changes into the thermodynamics of the system than changing ferromagnetic bonds. An additional feature which is absent in case of purely quantum antiferromagnetic sawtooth chain is the plateau at $M=1 / 4$ for $\eta<1$. In order to obtain a more satisfactory correspondence between numerical results for a purely quantum system and its mixed counterpart,one can consider a larger quantum cluster, containing three or more spins.

\section{Acknowledgements}

This work was partly supported by the grants CRDF-UCEP - 06/07 and ANSEF-1586-PS. The author would like to thank Oleg Derzhko for useful discussions and Lev Ananikian and Diana Antonosyan for help in preparing the figures. 


\title{
References
}

1. Cava R.J., Zandbergen H.W., Ramirez A.P., Takagi H., Chen C.T., Krajewski J.J., Peck W.F., Jr., Waszczak J.V., Meigs G., Roth R.S. Schneemeyer L.F., J. Solid State Chem., 1993, 104, 437.

2. Le Bacq O., Pasturel A., Lacroix C., Núñez-Regueiro M.D., Phys. Rev. B, 2005, 71, 014432.

3. Lau G.C., Ueland B.G., Freitas R.S., Dahlberg M.L., Schiffer P., Cava R.J., Phys. Rev. B, 2006, 73, 012413.

4. Kubo K., Phys. Rev. B, 1993, 48, 10552.

5. Nakamura T., Kubo K., Phys. Rev. B, 1996, 53, 6393.

6. Sen D., Shastry B.S., Walstedt R.E., Cava R., Phys. Rev. B, 1996, 53, 6401.

7. Nakamura T., Takada S., Phys. Lett. A, 1997, 225, 315.

8. Blundell S.A., Núñez-Regueiro M. D., Eur. Phys. J. B, 2003, 31, 453.

9. Richter J., Schulenburg J., Honecker A., Schnack J., Schmidt H.-J., J. Phys.: Condens. Matter, 2004, 16, S779.

10. Derzhko O., Honecker A., Richter J., Phys. Rev. B, 2007, 76, 220402(R).

11. Derzhko O., Richter J., Honecker A., Schmidt H.-J., Low Temp. Phys., 2007, 33, 745.

12. Richter J., Derzhko O., Honecker A., Int. J. Mod. Phys. B, 2008, 22, 4418.

13. Ohanyan V., Ananikian N., Phys. Lett., 2003, A 307, 76.

14. Ohanyan V., Ananikian N. - In: MATHEMATICAL PHYSICS Proceedings of the XI Regional Conference Tehran, Iran 2004. Eds. Rahvar S, Sadooghi N and Shojai F, World Scientific, 2005, p. 49-51.

15. Strečka J., Jaščur M., Hagiwara M., Minami K., Narumi Y., Kindo K., Phys. Rev. B, 2005, 72, 024459.

16. Strečka J., Jaščur M., J. Phys.: Condens. Matter, 2003, 15, 4519.

17. Čanová L., Strečka J., Jaščur M., J. Phys.: Condens. Matter, 2006, 18, 4967.

18. Strečka J., Čanová L., Lučivjanský T., Jaščur M., J. Phys.: Conf. Ser., 2009, 145, 012058.

19. Čanová L., Strečka J., Lučivjanský T. Preprint arXiv: 0903.4566, 2009.

20. Valverde J. S., Rojas O., de Souza S. M., J. Phys.: Condens. Matter, 2008, 20, 345208.

21. Antonosyan D., Bellucci S., Ohanyan V., Phys. Rev. B, 2009, 79, 014432.

22. Ohanyan V. Preprint arXiv: 0812.0127, 2008.

23. Pereira M. S. S., de Moura F. A. B. F., Lyra M. L., Phys. Rev. B, 2008, 77, 024402.

24. Pereira M. S. S., de Moura F. A. B. F., Lyra M. L., Phys. Rev. B, 2009, 79, 054427.

25. Baxter R. Exactly Solved Models in Statistical Mechanics. Academic Press, New York, 1982.

26. Oshikawa M., Yamanaka'M., Affleck I., Phys. Rev. Lett., 1997, 78, 1984.

\section{Антиферомагнітний пилкоподібний ланцюжок зі зв'язками Гайзенберга і Ізинга}

\author{
В. Оганян \\ ${ }^{1}$ Відділ теоретичної фізики, Єреванський державний університет, алея Манугяна 1, 0025 Єреван, \\ Вірменія \\ 2 Єреванський інститут фізики, бульвар Аліханяна 2, 0036 Єреван, Вірменія
}

Отримано 28 травня 2009 р., в остаточному вигляді - 18 червня 2009 р.

Розглядається пилкоподібний ланцюжок з парами $S=1 / 2$ спінів, що взаємодіють $3 X X Z$ взаємодією і розташовані на фрагменті кожного другого зубця пилки. Всі інші взаємодії взяті типу взаємодії Ізинга. Отримано точний статистико-механічний розв'язок моделі прямим методом матриці переносу. Розв'язок дозволяє отримати точні аналітичні вирази для всіх термодинамічних функцій моделі. Досліджено також властивості в основному стані і представлена відповідна фазова діаграма в основному стані.

Ключові слова: пилкоподібний ланцюжок, точні розв'язки, плато намагніченості

PACS: $75.10 . P q$ 
